\title{
Brachistosternus cepedai (Scorpiones: Bothriuridae), primer escorpión clasificado en peligro de extinción para el Desierto de Atacama: Fundamentos y consecuencias
}

\section{Brachistosternus cepedai (Scorpiones: Bothriuridae), the first scorpion to be designated as an endangered species in the Atacama Desert: Reasons and impacts}

\author{
Jaime Pizarro-Araya ${ }^{1,2 *}$ \& Andrés A. Ojanguren-Affilastro ${ }^{3}$ \\ ${ }^{1}$ Laboratorio de Entomología Ecológica, Departamento de Biología, Facultad de Ciencias, Universidad de La Serena, Casilla \\ 554, La Serena, Chile. \\ ${ }^{2}$ Instituto de Investigación Multidisciplinar en Ciencia y Tecnología, Universidad de La Serena, Casilla 554, La Serena, Chile. \\ ${ }^{3}$ División de Aracnología, Museo Argentino de Ciencias Naturales "Bernardino Rivadavia", Avenida Ángel Gallardo 470, \\ C1405DJR, Buenos Aires, Argentina. \\ *E-mail: japizarro@userena.cl
}

\begin{abstract}
RESUMEN
Se analizan los fundamentos por los cuales Brachistosternus cepedai (Scorpiones: Bothriuridae) ha sido considerado el primer escorpión en categoría de conservación en el Desierto de Atacama. Se discute sobre la sistemática y distribución particular de $B$. cepedai, así como las amenazas y posibles medidas para la conservación de esta especie endémica de un reducido sector del desierto costero chileno.
\end{abstract}

Palabras clave: Atacama, Brachistosternus, conservación, desierto costero, Scorpiones.

\begin{abstract}
We analyze the reasons for considering Brachistosternus cepedai (Scorpiones: Bothriuridae), the first scorpion to be included in a conservation category in the Atacama Desert. We also discuss the systematic position and restricted distribution of $B$. cepedai, as well as potential threats and conservation measures for this species, which is endemic to a reduced area of the Chilean coastal desert.
\end{abstract}

KeYwords: Atacama, Brachistosternus, conservation, coastal desert, Scorpiones.

\section{INTRODUCCIÓN}

La escorpiofauna de los desiertos costeros del norte de Chile es una de las más diversas del mundo (Agusto et al. 2006; Ojanguren-Affilastro et al. 2007a). El ambiente árido de la región y su temperatura templada constituyen condiciones especialmente favorables para este grupo de arácnidos (Agusto et al. 2006). Esto hace esperable una elevada diversidad y abundancia de representantes del grupo, tal como se observa en otros ambientes similares (Polis 1980, 1990; Nime et al. 2014). Sin embargo, es la particular orografía de la región, junto con la prolongada estabilidad de sus ambientes áridos en el tiempo, lo que más ha favorecido la notable diversificación de este grupo de artrópodos epigeos (Ceccarelli et al. 2016, 2017; Mongiardino-Koch et al. 2017; Pizarro-Araya \& Ojanguren-Affilastro en prensa).
Los factores ambientales predominantes en esta zona son la corriente fría de Humboldt al oeste, y la cordillera de los Andes al este. La corriente de Humboldt disminuye la temperatura del aire de la zona, bajando la humedad ambiental y reduciendo drásticamente las precipitaciones, pero, por otro lado, favorece la presencia de parches aislados de vegetación en sectores de la cordillera de la costa donde se condensa la niebla marina o camanchaca (Chand et al. 2010; Herrera et al. 2018). La cordillera de Los Andes impide la entrada de frentes húmedos desde el este, pero es el origen de los ríos que cruzan el desierto, que son alimentados por aguas de deshielo, y que configuran algunos de los elementos vicariantes más importantes del paisaje (Ceccarelli et al. 2017). Otros elementos vicariantes de importancia son los sistemas dunarios y los cordones montañosos internos, muchos de los cuales se conectan con 
la cordillera de los Andes, por lo que también han actuado como corredores para la fauna de altura (Ceccarelli et al. 2016). Debido a la latitud intermedia en que se encuentra esta zona $\left(28^{\circ} 29^{\prime} \mathrm{S}-29^{\circ} 20^{\prime} \mathrm{S}\right)$, y al relieve relativamente elevado que posee, toda esta área ha sido muy poco afectada por las glaciaciones y los posteriores aumentos del nivel del mar de los últimos 40 millones de años (Herrera et al. 2018). Es por esto que puede considerarse que las condiciones ambientales de la zona se han mantenido relativamente estables en este periodo, salvo por un gradual aumento de la aridez (Ceccarelli et al. 2017). Debido a todo lo antes mencionado, esta zona configura un mosaico ambiental notablemente complejo, con una cantidad altísima de elementos de artrópodos epigeos endémicos (Flores \& Pizarro-Araya 2006), lo que la hace particularmente vulnerable a cualquier perturbación por la actividad humana (Cárcamo et al. 2011).

En esta zona habitan solo dos familias de escorpiones: Caraboctonidae, representado por 1 género (Caraboctonus) y 1 especie descrita, C. keyserlingi Pocock 1893, y Bothriuridae, representado por 4 géneros (Bothriurus, Brachistosternus, Orobothriurus y Rumikiru) y alrededor de 30 especies descritas (Agusto et al. 2006; OjangurenAffilastro \& Pizarro-Araya 2014). En los últimos 15 años, investigadores del Laboratorio de Entomología Ecológica de la Universidad de La Serena, Chile (LEULS), junto con investigadores de la División de Aracnología del Museo Argentino de Ciencias Naturales Bernardino Rivadavia, Argentina (MACN-Ar), han realizado diversas expediciones con la finalidad de llevar a cabo relevamientos faunísticos en el norte y centro de Chile, que nos han permitido describir 22 de las casi 50 especies de escorpiones de Chile, lo que representa más del $40 \%$ de las especies conocidas del país (Ojanguren-Affilastro 2002, 2005; Ochoa et al. 2011; Ojanguren-Affilastro et al. 2007a, 2007b, 2010, 2011, 2012; Ojanguren-Affilastro \& Pizarro-Araya 2014), así como evaluar el estado de conservación de las mismas (PizarroAraya \& Ojanguren-Affilastro 2017). Es dentro de esta línea de trabajo que en el año 2016, se propuso por primera vez una especie de escorpión al Décimo Tercer Proceso de Clasificación de Especies Silvestres (Resolución Exenta $\mathrm{N}^{\mathrm{o}} 886 / 2016$, MMA) como especie protegida de Chile (que es también la primera en el Desierto de Atacama): Brachistosternus cepedai Ojanguren-Affilastro, Agusto, Pizarro-Araya \& Mattoni 2007 (Fig. 1), elemento endémico de las dunas costeras del norte de la Región de Coquimbo y del extremo sur de Atacama (Fig. 2). A mediados del año 2017, esta especie fue declarada "EN PELIGRO B1ab(iii)+2ab(iii)" por el Comité de Clasificación a través del Decreto Supremo No 6/2017 del Ministerio del Medio Ambiente (Constitución Política de la República de Chile; Ley N. ${ }^{\circ} 19.300$ ), debido a lo reducido de su hábitat, entre los $29^{\circ} 20^{\prime} \mathrm{S}$ y $28^{\circ} 29^{\prime} \mathrm{S}$ y entre los $71^{\circ} 29^{\prime} \mathrm{W}$ y $71^{\circ} 16^{\prime} \mathrm{W}$, y a la presión que la actividad humana está ejerciendo sobre el hábitat de la reducida población de Brachistosternus cepedai (Figs. 3a-d) (Pizarro-Araya \& Ojanguren-Affilastro 2017).

A continuación, brindamos una breve reseña sobre la sistemática y distribución de B. cepedai, así como los fundamentos utilizados para la propuesta. Además, discutimos posibles medidas para la conservación de esta y otras especies de la zona que se encuentran amenazadas por la modificación del ambiente.

\section{SisTEMÁTICA Y TAXONOMÍA}

Brachistosternus cepedai es una especie de pequeño tamaño, entre 36 y $66 \mathrm{~mm}$, (Ojanguren-Affilastro et al. 2007), que posee adaptaciones morfológicas para la vida en los sistemas dunarios de los que es endémica (Fig. 3a). Además del color amarillo claro similar al del sustrato en el que habita (Fig. 1), las mayores adaptaciones se dan en las estructuras de las patas, que son fundamentales para desplazarse en el suelo suelto de las dunas costeras casi carentes de vegetación (Fig. 3a). Estas incluyen una importante asimetría de las uñas y espolones tarsales, así como la presencia de una cantidad de setas tarsales mayor que la de otras especies de Brachistosternus. Debido a estas, y a otras adaptaciones a su medio, esta especie es una de las 3 que ha sido catalogada como ultrapsamófila, dentro de las casi 50 especies del género Brachistosternus (OjangurenAffilastro et al. 2016a).

Debido a la morfología externa y a la de sus hemispermatóforos, se consideró que Brachistosternus cepedai estaba estrechamente relacionada con Brachistosternus sciosciae Ojanguren-Affilastro 2002 (Ojanguren-Affilastro et al. 2007a; Ojanguren-Affilastro \& Ramírez 2009), una especie muy similar que habita en zonas cercanas, en los sistemas dunarios de Atacama (Ojanguren-Affilastro 2002). Sin embargo, un estudio filogenético basado en 3 marcadores nucleares, 2 marcadores mitocondriales y 116 caracteres morfológicos, reveló que ambas especies no están estrechamente relacionadas (Ojanguren-Affilastro et

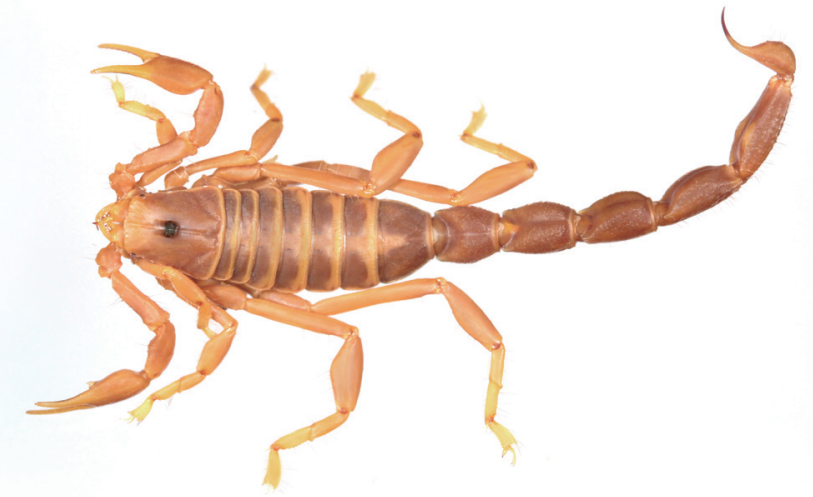

Figura 1. Habitus de Brachistosternus cepedai OjangurenAffilastro, Agusto, Pizarro-Araya \& Mattoni 2007 (Scorpiones: Bothriuridae) (§). / Habitus of Brachistosternus cepedai Ojanguren-Affilastro, Agusto, Pizarro-Araya \& Mattoni 2007 (Scorpiones: Bothriuridae) (đ) . 
al. 2016a). Brachistosternus cepedai forma un clado con representantes del género de zonas boscosas y de estepas arbustivas del centro y sur de Chile, siendo el único representante de su línea evolutiva dentro del género que ocupa este hábitat y que se encuentra en el norte de Chile. Por otro lado B. sciosciae pertenece a un clado diferente y se encuentra relacionada con especies de los desiertos del norte de Chile y sur del Perú. Debido a esto, se pudo concluir que la mayor parte de las similitudes morfológicas entre ambas especies responden a convergencias evolutivas por las fuertes presiones ambientales a las que fueron sometidas (Ceccarelli et al. 2017).
En una filogenia molecular datada, publicada un año más tarde (Ceccarelli et al. 2017), se pudo establecer que el ancestro de $B$. cepedai se separó del resto de las especies de su clado, que habitan más al sur, hace alrededor de 10 millones de años. Este periodo coincide con el comienzo del último y más abrupto levantamiento de los Andes, alrededor de $3000 \mathrm{~m}$ en 5 millones de años (Garzione et al. 2008; Ghosh et al. 2006), que intensificó las condiciones de aridez de la zona y promovió la aparición y el crecimiento de los ambientes dunarios sin vegetación donde habita $B$. cepedai.

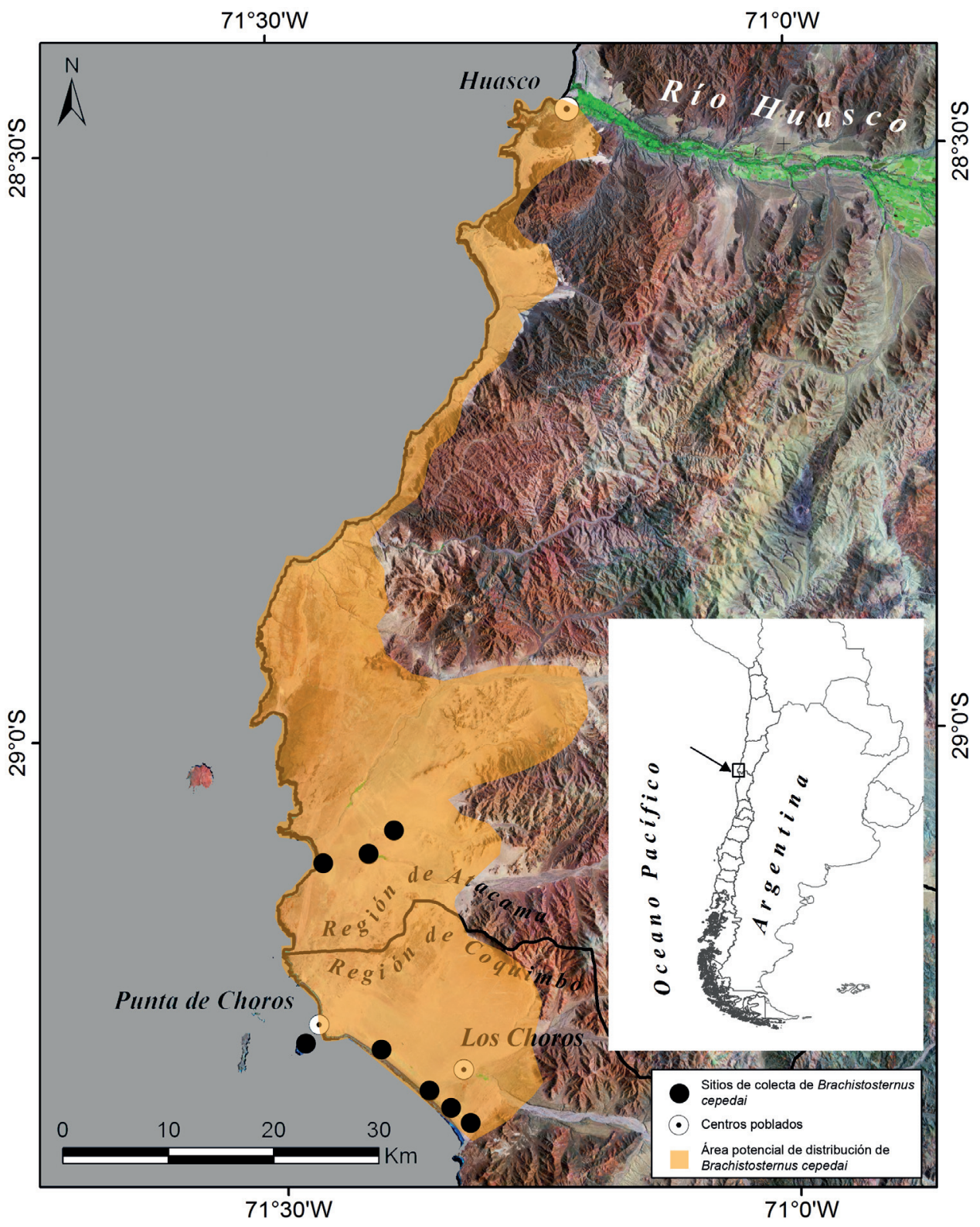

Figura 2. Mapa de distribución de Brachistosternus cepedai; se observa lo restringido de su distribución asociado a dunas costeras estabilizadas con vegetación nativa. / Distribution map of Brachistosternus cepedai; note the restricted distribution of this species, associated with coastal dunes stabilized by native vegetation. 

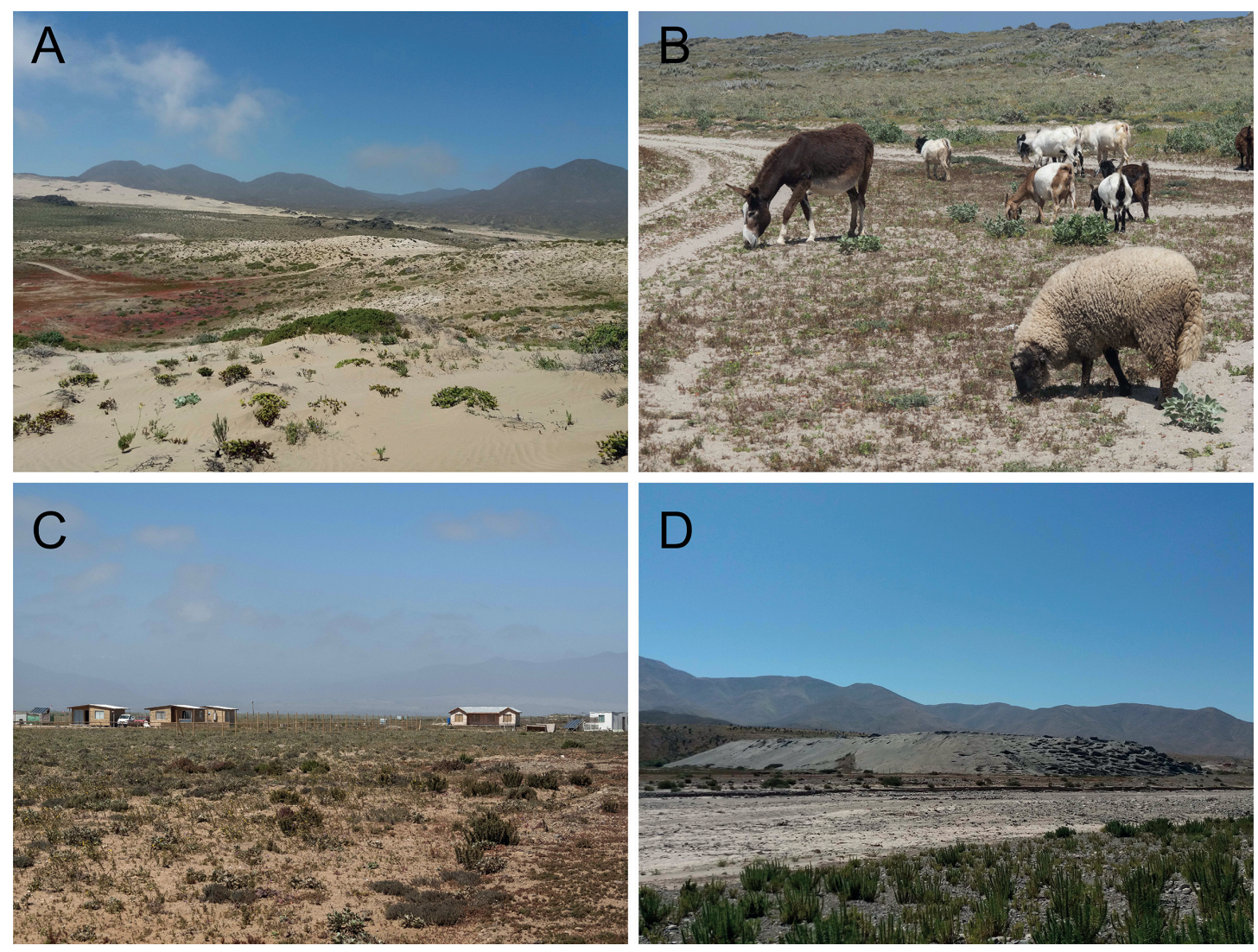

Figura 3. (A) Habitat dunario de Brachistosternus cepedai (Punta de Choros, Región de Coquimbo, Chile). (B-D) Amenazas de Brachistosternus cepedai: (B) Equus asinus, Capra hircus, Ovis orientalis aries, pastando sobre la vegetación nativa en las dunas costeras de Punta Choros (Región de Coquimbo, Chile). (C) Loteos de terrenos en los alrededores de las dunas costeras de Punta Choros (Región de Coquimbo, Chile). (D) Relaves mineros presentes en la Quebrada de Los Choros (Región de Coquimbo, Chile). / (A) Dune habitat of Brachistosternus cepedai (Punta de Choros, Coquimbo Region, Chile). (B-D) Threats to Brachistosternus cepedai; (B) Equus asinus, Capra hircus, and Ovis orientalis aries grazing on native vegetation in coastal dunes of Punta Choros (Coquimbo Region, Chile). (C) Land plots in coastal dunes of Punta Choros (Coquimbo Region, Chile). (D) Mine tailings at Quebrada de Los Choros (Coquimbo region, Chile).

\section{DiSTRIBUCIÓN Y HÁBITAT}

La distribución confirmada de Brachistosternus cepedai se reduce a las dunas costeras estabilizadas con y sin vegetación, que abarca desde Caleta Chañaral de Aceituno (Región de Atacama), hasta Punta Choros (Región de Coquimbo) en una zona de unos $50 \mathrm{~km}^{2}$ de largo y $c a 15 \mathrm{~km}^{2}$ de ancho (Fig. 2). El límite sur de distribución de esta especie está claramente marcado por las costas rocosas situadas al sur de Punta Choros y por los cordones montañosos costeros asociados con estos. En los sectores dunarios que se extienden más allá de estas costas rocosas, a partir de Punta Teatinos (Región de Coquimbo, Chile), B. cepedai es reemplazada por Brachistosternus cekalovici OjangurenAffilastro 2005. Hacia el norte, en cambio, la distribución de $B$. cepedai podría extenderse unos pocos kilómetros respecto de su distribución conocida, en un par de sectores dunarios costeros aislados ubicados al sur del río Huasco (en la Fig. 2 se indica el área potencial de distribución de
B. cepedai); sin embargo, aún no se tienen registros que confirmen su presencia en la zona. Al norte del río Huasco, B. cepedai es reemplazada por Brachistosternus sciosciae. En un estudio molecular filogeográfico de dos especies de escorpión del desierto costero chileno (Ceccarelli et al. 2017), se concluyó que los ríos permanentes que cruzan el desierto en dirección este-oeste, representan barreras efectivas para muchos artrópodos epígeos del desierto. Tal es el caso de varios escorpiones del género Brachistosternus que encuentran en el río Huasco su límite de distribución (Ceccarelli et al. 2017).

Brachistosternus cepedai también se encuentra presente en la isla Gaviota, perteneciente al archipiélago de Los Choros; sin embargo, no se encuentra en ninguna otra isla del archipiélago, ni en la cercana isla Chañaral (Reserva Nacional Pingüino de Humboldt) (Pizarro-Araya et al. 2014). Ceccarelli et al. (2017) demostraron que, hasta hace muy poco tiempo, existió flujo génico entre las poblaciones de 
escorpiones continentales y las que actualmente habitan las islas frente a Punta Choros (i.e., Reserva Nacional Pingüino de Humboldt). Esto se explica por la poca profundidad del fondo marino que separa estas islas del continente, que sólo alcanzó su nivel actual muy recientemente (Aguirre 1967). La ausencia de $B$. cepedai en la mayor parte de las islas de la zona, a pesar de que en ellas se encuentran presentes casi todos los elementos de la comunidad costera de escorpiones continentales, refleja la altísima especificidad del nicho ecológico utilizado por $B$. cepedai y la vulnerabilidad de esta especie.

Pizarro-Araya et al. (2014), analizaron la diversidad y composición estacional de la escorpiofauna del archipiélago de Los Choros (Región de Coquimbo), en este estudio se reportan los únicos antecedentes poblacionales para $B$. cepedai, de un total 258 escorpiones colectados, sólo el $2.3 \%$ correspondieron a $B$. cepedai. Cabe aclarar que el resto de las especies con las que es simpátrica, Brachistosternus roigalsinai (Ojanguren-Affilastro, 2002), Bothriurus coriaceus (Pocock, 1893) y Bothriurus dumayi (Cekalovic, 1974), se encuentran presentes en casi todos los ambientes del área, mientras que $B$. cepeda $i$ se encuentra limitado a los sectores dunarios, con y sin vegetación.

\section{VULNERABILIDAD Y AMENAZAS}

$\mathrm{Si}$ bien puede afirmarse que prácticamente todas las actividades antrópicas en el área donde habita $B$. cepedai afectan negativamente a esta especie, hemos identificado que los siguientes factores son los más relevantes en relación a su estado de conservación (En Peligro): 1) Pastoreo indiscriminado: uno de los mayores problemas es la falta de conciencia ambiental en relación al pastoreo indiscriminado y a la falta de manejo del ganado caprino (Capra hircus Linneo), ovino (Ovis orientalis aries Linneo) y equino (Equus asinus Linneo). Todavía no se tienen estimaciones definitivas de la reducción de la cobertura vegetal, sin embargo la observación empírica en el campo permite reconocer que las cabras han sido las causantes de la mayor disminución de la cobertura vegetal (Fig. 3b); 2) Extracción de vegetación nativa para su utilización como recurso energético (e.g., Eulychnia spp); 3) Desarrollo inmobiliario, ya que se trata de un borde costero que se ha visto afectado en los últimos años por la construcción de proyectos inmobiliarios de vivienda secundaria (Jorquera 2001) (Fig. 3c); 4) Megaminería a tajo abierto como el emprendimiento proyectado en sectores cercanos a Punta de Choros, debido a que las zonas de relave y la aspersión de ácido y otros compuestos químicos al medio natural necesariamente afectarán de manera negativa a la fauna epigea endémica del área (Fig. 3d). Tal como señalan Cárcamo et al. (2011), debería realizarse un análisis de los posibles efectos sinérgicos a escala espacial y temporal que podrían afectar a los distintos elementos de la biota, entre ellos los artrópodos epigeos.
PRopuestas DE MEDIDAS DE MITIGACIÓN

En casos de especies consideradas En Peligro, como el de Brachistosternus cepedai, es fundamental elaborar medidas de mitigación que permitan evitar o reducir los efectos ecológicos negativos identificados para sus poblaciones, tales como la destrucción, fragmentación y degradación del hábitat; considerando, además, la alta especialización de hábitat de este taxón (Bustamante et al. 2009).

Cuando nos basamos en el criterio de categoría de conservación, y en el caso particular de Brachistosternus cepedai, que fue declarada "EN PELIGRO", las medidas propuestas se deben centrar en la especie y en las acciones tendientes a la protección de estas poblaciones y su hábitat (SAG, 2004). De acuerdo a lo anterior, según este enfoque, las medidas de mitigación deben ser diseñadas e implementadas con el objetivo de preservar, conservar o mejorar el conjunto de condiciones ambientales que permiten la permanencia y existencia de las especies.

Actualmente el área donde habita $B$. cepedai no está incluida en ningún área protegida del sistema SNASPE (Sistema Nacional de Áreas Silvestres Protegidas del Estado); sin embargo, el área de Punta Choros donde existen extensos sectores dunarios en los que está presente esta especie, se encuentra contigua a la Reserva Nacional Pingüino de Humboldt. La ampliación de esta reserva al área continental permitiría proteger, no sólo a $B$. cepedai, sino también a toda la fauna epigea de estos sistemas dunarios que aún no ha sido estudiada (Pizarro-Araya et al. 2012; Jerez et al. 2015).

\section{CONCLUSIONES}

Los ambientes áridos y semiáridos del mundo pueden parecer engañosamente desprovistos de vida; sin embargo, muchos de estos poseen una notable biodiversidad, y un alto grado de endemismo. Los desiertos costeros del norte de Chile son uno de los mejores ejemplos de ello, ya que representan un hotspot de diversidad y endemismos para muchos grupos de artrópodos (Roig-Juñent et al. 2006). Tal es el caso de los escorpiones del género Brachistosternus, los que en esta zona tienen sus puntos de mayor diversidad filogenética y de mayor especificidad evolutiva (Ceccarelli et al. 2016; Ojanguren-Affilastro et al. 2016b, Mongiardino-Koch et al . 2017). Debido a esto, y a la cada vez mayor presión que ejerce el hombre sobre el ambiente, deben desarrollarse estrategias de conservación específicas que contemplen todos los aspectos de la problemática en cuestión y que tengan en cuenta a todos los actores involucrados.

El caso planteado de B. cepedai puede servir, además, como ejemplo para implementar estrategias de conservación de artrópodos como herramientas dentro de la conservación de ambientes amenazados, ya que debido a su notable especificidad de nicho ecológico muchos escorpiones de 
este grupo pueden llegar a constituirse como excelentes bioindicadores.

\section{AGRADECIMIENTOS}

Agradecemos a CONAF por la ayuda en los permisos y facilidades para trabajar en la Reserva Nacional Pingüino de Humboldt (Proyectos No 18/2011 y Nº 006/2014). Hacemos extensión a todos los colegas y amigos que han participado en las expediciones que hemos realizados al desierto costero chileno: Fermín M. Alfaro, Pablo Agusto, Juan Enrique Barriga, Ricardo Botero-Trujillo, Martín J. Ramírez, Hernán A. Iuri, Tío Dogui (Punta de Choros), Patricio Ortiz (Aljavig, Chañaral de Aceituno), José Loyola (Punta Cachos), Alberto Castex (Monoclope.cl), Paola VargasTalciani, Cristian Rivera (Isla Chañaral, CONAF, Atacama), Paula Martínez, Carla Louit, Diego Morales y Pablo Arróspide (Islas Choros y Damas, CONAF, Coquimbo). El presente estudio fue financiado por los proyectos DIDIULS PR17121/VACDDI001 de la Universidad de La Serena, La Serena, Chile (JPA) y PIP 11220150100672 CO (AAOA).

\section{REFERENCIAS}

Aguirre, L. 1967. Geología de las islas Choros, Damas y de Punta Choros, Provincia de Coquimbo. Revista Minerales (Chile) 22: 73-83.

Agusto, P., Mattoni, C.I., Pizarro-Araya, J., Cepeda-Pizarro, J., López-CortÉs, F. 2006. Comunidades de escorpiones (Arachnida: Scorpiones) del desierto costero transicional de Chile. Revista Chilena de Historia Natural 79: 407-421.

Bustamante, R.O., Oporto, A., Moraga, S., de La Barrera, F., Sepúlveda G., Moreira, D. 2009. Informe sobre mitigación de impacto ambiental en Fauna Silvestre: Rescate y Relocalización. SAG-Universidad de Chile, Santiago, Chile. 73 pp.

Cárcamo, P.F., Cortés, M., Ortega, L., Squeo, F.A., Gaymer, C.F. 2011. Crónica de un conflicto anunciado: Tres centrales termoeléctricas a carbón en un hotspot de biodiversidad de importancia mundial. Revista Chilena de Historia Natural 84: 171-180.

Ceccarelli, F.S., Ojanguren-Affilastro, A.A., Ramírez, M.J., OchoA, J.A., Mattoni, C.I., Prendini, L. 2016. Andean uplift drives diversification ofthe bothriurid scorpion genus Brachistosternus. Journal of Biogeography 43: 1942-1954.

Ceccarelli, F.S., Pizarro-Araya, J., Ojanguren-Affilastro, A.A. 2017. Phylogeography and population structure of two Brachistosternus species (Scorpiones: Bothriuridae) from the Chilean coastal desert - The perils of coastal living. Biological Journal of the Linnean Society 120: 75-89.

Chand, D., Hegg, D.A., Wood, R., Shaw, G.E., Wallace, D., Covert, D.S. 2010. Source attribution of climatically important aerosol properties measured at Paposo (Chile) during VOCALS. Atmospheric Chemistry and Physics 10:
10789-10801.

Flores, G.E., Pizarro-Araya, J. 2006. The Andes mountain range uplift as a vicariant event in the Pimeliinae (Coleoptera: Tenebrionidae) in Southern South America. Cahiers Scientifiques 10: 95-102.

Herrera, C., Gamboa, C., Custodio, E., Jordan, T., Godfrey, L., Jodar, J., Luque, J.A., Vargas, J., SÁez, A. 2018. Groundwater origin and recharge in the hyperarid Cordillera de la Costa, Atacama Desert, northern Chile. Science of the Total Environment 624: 114-132.

Ghosh, P., Garzione, C.N., Eiler, J.M. 2006. Rapid uplift of the Altiplano revealed through $13 \mathrm{C}-18 \mathrm{O}$ bonds in paleosol carbonates. Science 311: 511-515.

Garzione, C.N., Hoke, G.D., Libarkin, J.C., Withers, S., MacFadden, B., Eiler, J., Ghosh, P., Mulch, A. 2008. Rise of the Andes. Science 320: 1304-1307.

Jerez, V., Zúñiga-Reinoso, A., Muñoz-Escobar, C., PizarroArAYA, J. 2015. Acciones y avances sobre la conservación de insectos en Chile. Gayana 79(1): 1-3.

Jorquera, C. 2001. La agricultura regional y el deterioro de la vegetación nativa: Una visión actualizada. En: Squeo, F.A., Arancio, G., Gutiérrez, J.R. (eds.) Libro rojo de la flora nativa y de los sitios prioritarios para su Conservación: Región de Coquimbo: 239-251. Ediciones Universidad de La Serena, La Serena, Chile.

Mongiardino-Koch, N., Ceccarelli, F.S., Ojanguren-Affilastro, A.A., Ramírez, M.J. 2017. Discrete and morphometric traits reveal contrasting patterns and processes in the macroevolutionary history of a clade of scorpions. Journal of Evolutionary Biology 30(4): 814-825.

Nime, M.F., Casanoves, F., Mattoni, C.I. 2014. Scorpion diversity in two different habitats in the Arid Chaco, Argentina. Journal of Insect Conservation 18(3): 373-384.

Ochoa, J.A., Ojanguren-Affilastro, A.A., Mattoni, C.I., Prendini, L. 2011. Systematic revision of the Andean scorpion genus Orobothriurus Maury 1976 (Bothriuridae), with discussion of the altitude record for scorpions. Bulletin of the American Museum of Natural History 359: 1-90.

OJanguren-Affilastro, A.A. 2002. Nuevos aportes al conocimiento del género Brachistosternus en Chile, con la descripción de dos nuevas especies (Scorpiones, Bothriuridae). Boletín de la Sociedad de Biología de Concepción (Chile) 73: 37-46.

Ojanguren-Affilastro, A.A. 2005. Notes on the genus Brachistosternus (Scorpiones, Bothriuridae) in Chile, with the description of two new species. Journal of Arachnology 33: 175-192.

Ojanguren-Affilastro, A.A., Ramírez, M.J. 2009. Phylogenetic analysis of the scorpion genus Brachistosternus (Arachnida, Scorpiones, Bothriuridae). Zoologica Scripta 38: 183-198.

Ojanguren-Affilastro, A.A., Pizarro-Araya, J. 2014. Two new scorpion species from Paposo, in the Coastal desert of Taltal, Chile (Scorpiones, Bothriuridae, Brachistosternus). Zootaxa 3785(3): 400-418.

Ojanguren-Affilastro, A.A., Pizarro-Araya, J., Prendini, L. 2011. New data on Chilean Urophonius Pocock 1893, with description o a new species. American Museum Novitates 3725: 1-44 pp.

Ojanguren-Affilastro, A. A., Ochoa, J.A., Mattoni, C., Prendini, L. 2010. Systematic revision of the granulatus group of 
Urophonius Pocock, 1893 (Scorpiones, Bothriuridae), with description of a new species from central Chile. American Museum Novitates 3695: 1-40.

Ojanguren-Affilastro, A.A., Ochoa, J.A., Mattoni, C., Prendini, L. 2012. Rumikiru, n. gen. (Scorpiones, Bothriuridae). A new Scorpion genus from the Atacama Desert. American Museum Novitates 3731: 1-43.

Ojanguren-Affilastro, A.A., Agusto, P. Pizarro-Araya, J., Mattoni, C.I. 2007a. Two new scorpion species of genus Brachistosternus (Scorpiones: Bothriuridae) from northern Chile. Zootaxa 1623: 55-68.

Ojanguren-Affilastro, A.A., Mattoni, C.I., Prendini, L. 2007b. The genus Brachistosternus (Scorpiones: Bothriuridae) in Chile, with descriptions of two new species. American Museum Novitates 3564: 1-44.

Ojanguren-Affilastro, A.A., Mattoni, C.I., OchoA, J.A., Ramírez, M.J., Ceccarelli, F.S., Prendini, L. 2016a. Phylogeny, species delimitation and convergence in the South American bothriurid scorpion genus Brachistosternus Pocock 1893: Integrating morphology, nuclear and mitochondrial DNA. Molecular Phylogenetics and Evolution 94: 159-170.

Ojanguren-Affilastro, A.A., Botero-Trujillo, R., Castex, A., Pizarro-ArayA, J. 2016b. Biological aspects of the genus Brachistosternus (Bothriuridae) in the Atacama Desert (Chile), with the description of a new type of pedipalp macroseta. Gayana 80(2): 162-167.

Pizarro-Araya, J., Ojanguren-Affilastro, A.A. En prensa. El orden Scorpiones en Chile: estado del conocimiento. En: Canals, M., Aguilera M., Taucare, A. (eds.) Proyecto Arañas comunes de Chile. Ediciones Universidad de Chile, Santiago, Chile.
Pizarro-Araya, J., Ojanguren-Affilastro, A.A. 2017. Brachistosternus cepedai Ojanguren-Affilastro, Agusto, Pizarro-Araya \& Mattoni 2007. En: $13^{\circ}$ Proceso de Clasificación de Especies (Resolución exenta No ${ }^{\circ} 1150$ MMA). Ministerio del Medio Ambiente, Chile. EN PELIGRO EN B1ab(iii)+2ab(iii) http://www.mma. gob.cl/clasificacionespecies/fichas 13 proceso/fichas PAC_13RCE/Brachistosternus_cepedai_PAC_13RCE.pdf

Pizarro-Araya, J., Vergara, O.E., Flores, G.E. 2012. Gyriosomus granulipennis Pizarro-Araya \& Flores 2004 (Coleoptera: Tenebrionidae) un caso extremo a conservar. Revista Chilena de Historia Natural 85(3): 345-349.

Pizarro-Araya, J., Agusto, P., López-Cortés, F., OjangurenAffilastro, A.A., Briones, R., Cepeda-Pizarro, J. 2014. Diversidad y composición estacional de la escorpiofauna (Arachnida: Scorpiones) del archipiélago Los Choros (Región de Coquimbo, Chile). Gayana 78(1): 46-56.

Polis, G.A. 1980. Seasonal patterns and age-specific variation in the surface activity of a population of desert scorpions in relation to environmental factors. Journal of Animal Ecology 49:1-18.

Polis, G.A. 1990. The biology of scorpions. Standford University Press, Standford.

Roig-Juñent, S., Domínguez, M.C., Flores, G.E., Mattoni, C. 2006. Biogeographic history of South American arid lands: A view from its arthropods using TASS analysis. Journal of Arid Environments 66(3): 404-420.

SAG. 2004. Medidas de mitigación de impactos ambientales en fauna silvestre. División de Protección de los Recursos Naturales Renovables Subdepartamento de Gestión Ambiental. Santiago, Chile. 180 pp.

Recibido: 22.11.2017

Aceptado: 16.05.2018 